\title{
Classification and Location of Atrial Arrhythmic Mechanisms With Body Surface Potential Mapping
}

\author{
Victor G Marques $^{1}$, Miguel Rodrigo ${ }^{2}$, María S Guillem ${ }^{2}$, João Salinet ${ }^{1}$ \\ ${ }^{1}$ Federal University of ABC, São Bernardo do Campo, Brazil \\ 2 ITACA Institute, Universitat Politècnica de València, Spain
}

\begin{abstract}
Atrial tachycardia (AT), flutter (AFL) and fibrillation $(A F)$ are very common cardiac arrhythmias and are driven by localized sources that can be ablation targets. Noninvasive body surface potential mapping (BSPM) can be useful for early diagnosis and ablation planning. We aimed to automatically classify and locate the arrhythmic mechanisms behind AT, AFL and AF using BSPM features. 19 simulations of 567-lead BSPMs were used to obtain dominant frequency (DF) maps from which features reflecting the spatial distribution of DFs and the spectral organization were extracted. Rotational activity was tracked based on singularity points in phase maps; features were extracted to reflect its spatio-temporal stability. The torso was divided in 4 quadrants to assess the spatial distribution of the features. Random forest and least-square based algorithms were used to classify the arrhythmias and their mechanisms' location, respectively. The analyses were reproduced in different layouts (252 to 12 leads). The arrhythmic mechanisms and their locations were classified with $72.0 \%$ and $73.9 \%$ balanced accuracy, respectively. Accuracy was similar along all lead layouts for arrhythmia classification but decreased for mechanism location. Classification of AT, AFL and AF and their mechanisms' location was feasible based on BSPM features reflecting their basic electrophysiological characteristics.
\end{abstract}

\section{Introduction}

Focal atrial tachycardia (AT), atrial flutter (AFL) and atrial fibrillation (AF) are among the most common supraventricular tachyarrhythmias (SVTs), a group of very prevalent cardiac arrhythmias and increase morbidity and mortality due to a higher risk of thromboembolic events and of developing other cardiac diseases, representing significant costs for the health services [1]. These arrhythmias are driven by localized sources, which for certain groups can be targeted in ablation therapy to restore sinus rhythm $[1,2]$ : AT is maintained by ectopic foci [1], AFL by macro- reentrant circuits around anatomic structures [1], and $\mathrm{AF}$, which is the most complex of these arrhythmias, has been shown to be maintained by ectopic foci, re-entrant circuits and functional rotors, which appear preferably when structural and/or functional atrial remodeling occurs [3]

Identification of the driving mechanisms and their location is crucial for further understanding these arrhythmias and for achieving higher rates of success in ablation therapies, especially for AF patients [4]. This is usually performed via an invasive electrophysiological study, which is technically complex, time-consuming and poses considerable risks to the patients. Non-invasive techniques, such as body surface potential mapping (BSPM), present reduced costs and risks to the patients, while providing relevant clinical information, thus being useful for early diagnosis, ablation planning and follow-up of patients [5].

Detecting regions driving AT and typical AFL is relatively straightforward, using analysis of activation times to locate the site of earliest activation or reentrant circuit, respectively [2]. In atypical AFL and AF, the complexity of the underlying electrophysiological patterns reduces the accuracy of direct analysis of activation times [6]. Analyses based in dominant frequency (DF) or phase have been used in recent years as a means to find periodicity measurements and rotational patterns both in invasive and noninvasive signals [4, 6-8], allowing for the location of driving mechanisms and improved ablation outcomes $[4,8]$.

In this study, we used realistic computer models to investigate AT, AFL and AF from the non-invasive BSPM perspective, applying DF and phase analysis to extract features characterizing the arrhythmic driving mechanisms and their location. Simple pattern recognition algorithms were applied for classification of these parameters.

\section{Methods}

A realistic three-dimensional model of the atrial anatomy was used to simulate the electrical behavior of the left and right atria (LA and RA, respectively) in arrhythmic conditions. 19 simulations represented arrhythmias originated by three distinct mechanisms: AT (4 sim- 

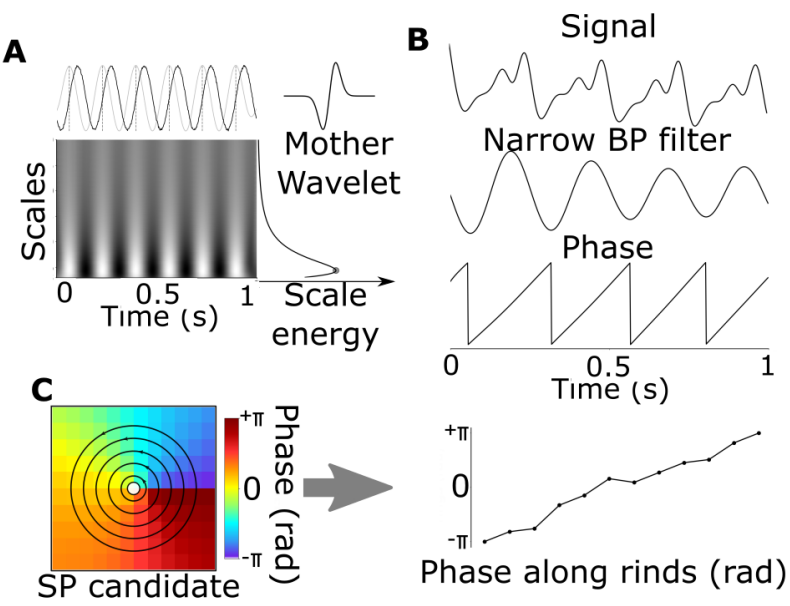

Figure 1. Schematic of the methodology. A: wavelet method for DF detection; B: Phase calculation in BSPM signals; $\mathrm{C}$ : phase progression analysis for SP detection

ulations) driven by an ectopic focus; AFL (4 simulations) driven by a macro-reentrant circuit; AF (11 simulations) driven by functional rotors [9]. The highest peak in Welch periodograms was used to determine dominant frequencies (DFs) inside the atria [9]; the highest dominant frequency (HDF) in the atria was defined as the driving frequency [8].

BSPMs were obtained by solving the forward problem with the boundary element method, resulting in 771 data points referenced to the Wilson central terminal and sampled at $f_{s}=500 \mathrm{~Hz}$ [9], of which 567 points were selected to represent leads of a realistic measurement, excluding points inside the waist, neck or arms. White Gaussian noise was added to the BSPM signals with a signalto-noise ratio (SNR) of $60 \mathrm{~dB}$ [9]. The 3D coordinates of the BSPM nodes in the models were mapped to a $2 \mathrm{D}$ configuration by projecting their positions into a cylinder wall and unwrapping it with the left side of the torso in the center of the image. The signals were interpolated into a 30 by 65 grid using cubic splines [7].

DFs on BSPM signals were estimated with a method based on continuous wavelet transform (CWT) [10]. Peaks detected in the outcomes of a CWT performed with a negative first-order Gaussian wavelet match patterns similar to singularities [10], such as the sharp positive deflections associated with depolarization wavefronts in BSPM signals [11]. CWT was applied for each lead along 40 linearly spaced scales in the pseudo-frequency range of 3 to $30 \mathrm{~Hz}$ (Figure 1A). Peak detection was performed in the scale with maximum energy in this range after low-pass filtering it at $30 \mathrm{~Hz}$ (4th order Butterworth) to avoid double counting. The average of the cycle lengths (CL) was determined from the intervals between the detected peaks and used for the DF calculation $(D F=1 / C L)$.

DF maps were generated by displaying the color coded
DF values in the correspondent lead positions, and were used to approximate the driver mechanism frequencies with the HDF from BSPMs $\left(\mathrm{HDF}_{B S P M}\right)$. A spatial mask ignoring $6 \%$ of the highest DF values was implemented to avoid small harmonic regions present mainly in AT and AFL maps. HDF regions $(|D F-H D F| \leq 1 \mathrm{~Hz})$ on the torso were segmented and used for feature extraction.

A narrow band-pass filter (4th order Butterworth) was applied on the BSPM leads around the estimated atrial HDFs $( \pm 1 \mathrm{~Hz})$ to stabilize the atrial activity in the BSPM, especially for AF (Figure 1B) [12]. The signals were downsampled to $128 \mathrm{~Hz}$ to decrease computation time and phase was obtained using the Hilbert transform [12].

Rotors were detected through phase singularity points (SPs), defined as points around which all phases converge, by detecting the discontinuity in phase maps corresponding to the sharp transition between $+\pi$ and $-\pi$ [9]. Canny edge detection was used to detect these transitions in phase maps [9] and the endpoints of the edges were considered SP candidates. The selection of the true SPs among the candidates is made based on the phase progression along five rings (radii 2 to $10 \mathrm{~cm}$ ) around them (Figure 1C, left). The phase progression of a SP (Figure 1C, right) should attend three criteria in at least two of the rings: the phase progresses in an range of at least $\pi$, the progression is at least $60 \%$ ordered and there are no phase leaps $\geq \pi$ [9].

Features reflecting the spatio-temporal distribution of the SPs were obtained from filament and heatmaps (HMs). A filament is defined as the connection of the SPs in phase maps along subsequent time instants, around which at least one full cycle of rotation was sustained. HMs are the histogram of the SPs belonging to filaments along time.

The signals were classified by pattern recognition algorithms with respect to the underlying arrhythmia (AT, AFL or AF) and the location of their driver in the heart (LA or RA). Different groups of features were used for each classification task: for the arrhythmia classification, 11 features were extracted from DF maps, filaments and HMs for each simulation: ratio between mean DF and HDF, number and average size of HDF regions, DF range, mean and IQR of the organization index (OI) on each lead [13], number, mean duration and median spatial displacement of filaments (calculated as the median area of the bounding boxes of the SP clusters originated by each individual filament), number and median density of SP clusters.

The classifier was organized in two layers: the first discriminates between AF and the remaining SVTs, whereas the second layer discriminates between AT and AFL. Each layer is composed by a random forest model trained using 40 decision trees of maximum depth of 2 . In each node, one of 3 randomly selected features was selected to split the dataset using axis-aligned linear discriminators based on the resulting information gain [14]. The average results 
from the trees determines the algorithm's prediction.

For the mechanism location classification, the torso was divided into 4 overlapping quadrants (left vs. right and front vs. back). 3 features were extracted from each quadrant, resulting in a total of 12 features: percentage of the region with $\left|D F-\mathrm{HDF}_{B S P M}\right| \leq 1 \mathrm{~Hz}$, average OI, and percentage of SPs. The localization of the mechanisms was classified with respect to the atrium in which it was located (LA vs. RA) with a simple least-squares algorithm.

Leave-one-out cross validation was used to test the generalization of the classification approaches and results were evaluated based on confusion matrices and balanced accuracy. The analyses were reproduced for subsets of the original lead layout to assess the stability of the features to lower resolution scenarios: 256 [15], 127[5], 67[7], 64[16], 32, 16 , and 12 [17].

\section{Results}

Fig. 2A shows the confusion matrix for the arrhythmia classification. Accuracy was highest for AF (90.1\%), followed by AT (75.0\%), and AFL (50\%). The overall balanced accuracy was $72.0 \%$. While AF classification reflected its distinct behavior in the DF and phase analyses (Fig. 3, bottom), distinguishing AT from AFL with the proposed features was more challenging. This happens due to the similar DF distributions (very uniform with small harmonic regions, Fig. 3A) and SP propagation, as most AT simulations presented at least one stable filament, though less spatially localized than those observed in AFL (Fig. 3B).

Fig. 2B shows the results for the classification along different lead layouts. AF segments are classified with high accuracy in all layouts, whereas for AT and AFL lower accuracies and greater variability is observed, mainly due to the limited number of samples.

Fig. 2C displays the confusion matrix for the driver location classification using the least squares algorithm. Balanced accuracy value of $73.9 \%$ was achieved by this approach (70\% and $77.8 \%$ for the LA and RA, respectively). Fig. 2D shows the effect of reducing the number of leads in the classification of the driving mechanism location. The resulting accuracies are progressively lower with the reduction in the number of leads.

\section{Discussion and Conclusion}

In this work, features extracted from BSPM DF maps, filaments and HMs allowed the arrhythmias classification with good balanced accuracy, being able to distinguish AF in the vast majority of the tested simulations, reflecting its particular behavior detected by more heterogeneous DF distributions and shorter, moving filaments. The more similar patterns observed between AT and AFL indicate that
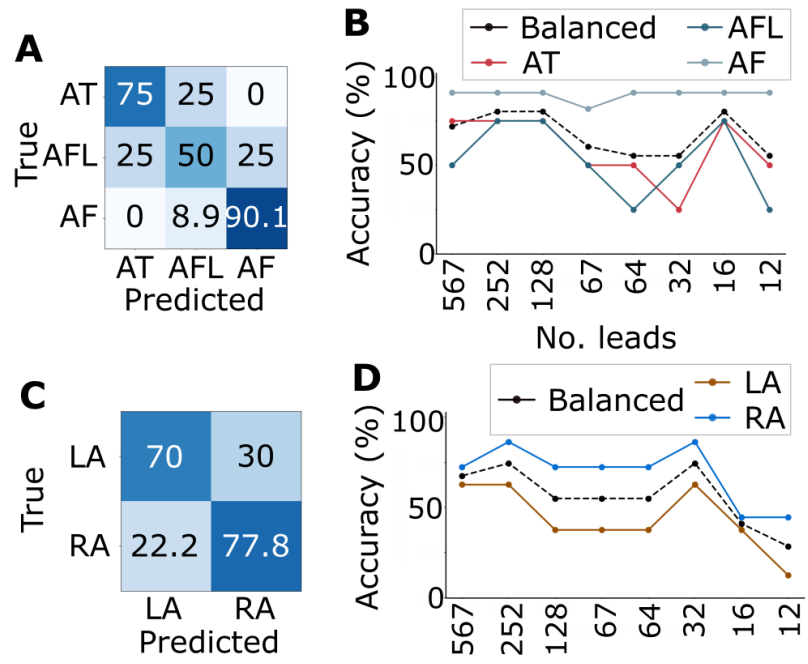

No. leads

Figure 2. Confusion matrices for the arrhythmia classification (A) and the mechanism location (B). Accuracies along layouts are respectively shown in $\mathrm{C}$ and $\mathrm{D}$.

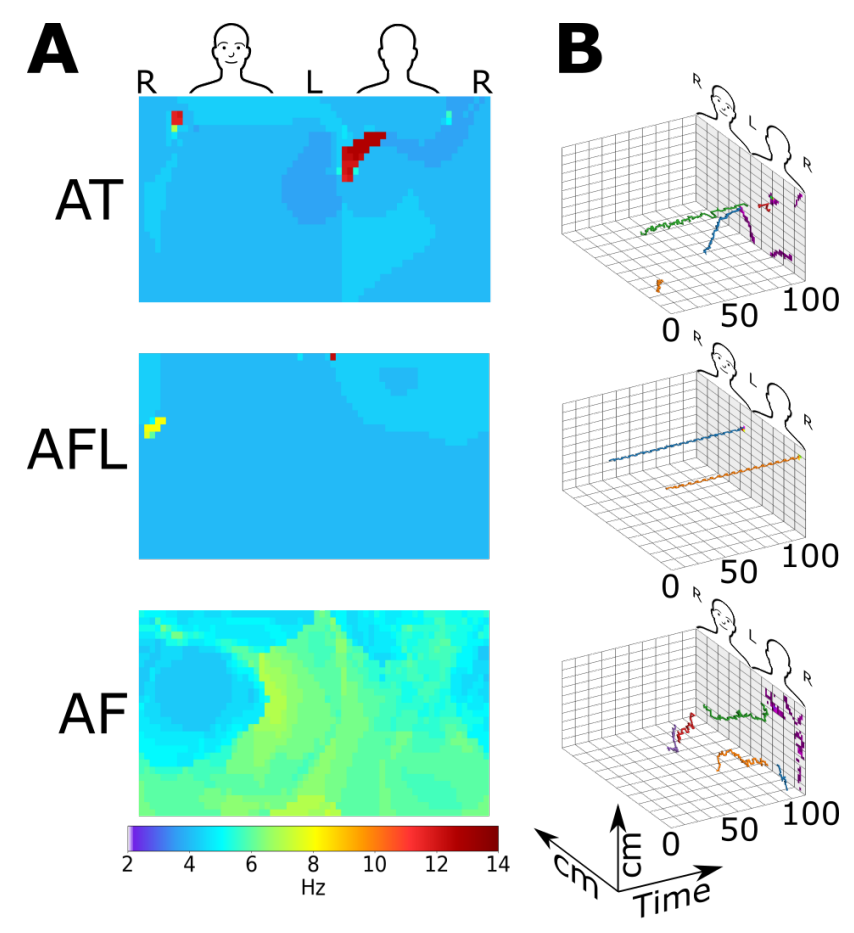

Figure 3. Typical examples of DF maps (A) and filaments with HMs (B), for each arrhythmia

adding more features reflecting the specific characteristics of these arrhythmias, such as detecting breakthroughs in AT or analyzing the polarity of $\mathrm{P}$ waves, may improve the classification outcomes. The results for the localization of mechanisms highlight the need of incorporating additional metrics to achieve more reliable results with BSPM. The 
poorer performance in lower resolution layouts highlight the relevance of analyzing critically results obtained with a limited number of leads. Additional classification algorithms, including support vector machines and k-nearest neighbors, were also applied to the data but had worse performances than those presented here, both for arrhythmia and driver location classification.

SVT detection based on non-invasive techniques is highly desirable, especially for AF due to its complexity. Different from the previous studies in the literature, the present work could classify the three most important SVTs and their driving mechanism location on the atria with relatively simple machining learning algorithms. The classification was based on relevant arrhythmia biomarkers extracted from maps reflecting the basic electrophysiological mechanisms maintaining the arrhythmias, generated with an unified methodology and using high density BSPM signals, contributing to the current knowledge of non-invasive electrophysiological analysis of atrial arrhythmias. This is the first study to the extent of the authors' knowledge to classify AT, AFL and AF and their driving mechanisms' locations using BSPM data and applying an unified methodology.

\section{Acknowledgements}

This study was supported in part by grants from São Paulo Research Foundation (2017/19775-3), Instituto de Salud Carlos III FEDER (Fondo Europeo de Desarrollo Regional PI17/01106) and Generalitat Valenciana Grants (AICO/2018/267).

\section{References}

[1] Page RL, Joglar JA, Caldwell MA, Calkins H, Conti JB, Deal BJ, et al. $2015 \mathrm{acc} / \mathrm{aha} / \mathrm{hrs}$ guideline for the management of adult patients with supraventricular tachycardia. J Am Coll Cardiol 2016;67(13):e27-e115.

[2] Issa ZF, Miller JM, Zipes DP. Clinical arrhythmology and electrophysiology: a companion to Braunwaldś heart disease. Elsevier Health Sciences, 2009.

[3] Jalife J, Berenfeld O, Mansour M. Mother rotors and fibrillatory conduction: a mechanism of atrial fibrillation. Cardiovasc Res 2002;54(2):204-216.

[4] Narayan SM, Krummen DE, Shivkumar K, Clopton P, Rappel WJ, Miller JM. Treatment of atrial fibrillation by the ablation of localized sources: CONFIRM trial. J Am Coll Cardiol 2012;60(7):628-636.

[5] Vanheusden FJ, Chu GS, Li X, Salinet J, Almeida TP, Dastagir N, et al. Systematic differences of non-invasive dominant frequency estimation compared to invasive dominant frequency estimation in atrial fibrillation. Comput Biol Med 2019;104:299-309.

[6] Ng J, Kadish AH, Goldberger JJ. Effect of electrogram characteristics on the relationship of dominant frequency to atrial activation rate in atrial fibrillation. Heart Rhythm 2006;3(11):1295-1305.

[7] Guillem MS, Climent AM, Millet J, Arenal n, FernándezAvilés F, Jalife J, et al. Noninvasive localization of maximal frequency sites of atrial fibrillation by body surface potential mapping. Circ Arrhythm Electrophysiol 2013; 6(2):294-301.

[8] Atienza F, Almendral J, Ormaetxe JM, Moya n, MartínezAlday JD, Hernández-Madrid A, et al. Comparison of radiofrequency catheter ablation of drivers and circumferential pulmonary vein isolation in atrial fibrillation: a noninferiority randomized multicenter radar-af trial. J Am Coll Cardiol 2014;64(23):2455-2467.

[9] Rodrigo M, Climent AM, Liberos A, Fernández-Avilés F, Berenfeld O, Atienza F, Guillem MS. Technical considerations on phase mapping for identification of atrial reentrant activity in direct-and inverse-computed electrograms. Circ Arrhythm Electrophysiol 2017;10(9):e005008.

[10] Marques VG, Rodrigo M, Guillem MS, Salinet J. A robust wavelet-based approach for dominant frequency analysis of atrial fibrillation in body surface signals. Physiol Meas 2020;

[11] Guillem MS, Climent AM, Castells F, Husser D, Millet J, Arya A, Piorkowski C, Bollmann A. Noninvasive mapping of human atrial fibrillation. J Cardiovasc Electrophysiol 2009;20(5):507-513.

[12] Rodrigo M, Guillem MS, Climent AM, Pedrón-Torrecilla J, Liberos A, Millet J, Fernández-Avilés F, Atienza F, Berenfeld O. Body surface localization of left and right atrial high-frequency rotors in atrial fibrillation patients: a clinical-computational study. Heart Rhythm 2014; 11(9):1584-1591.

[13] Everett IV TH, Moorman JR, Kok LC, Akar JG, Haines DE. Assessment of global atrial fibrillation organization to optimize timing of atrial defibrillation. Circulation 2001; 103(23):2857-2861.

[14] Criminisi A, Shotton J. Decision forests for computer vision and medical image analysis. Springer Science \& Business Media, 2013.

[15] Haïssaguerre M, Hocini M, Denis A, Shah AJ, Komatsu Y, Yamashita S, et al. Driver domains in persistent atrial fibrillation. Circulation 2014;130(7):530-538.

[16] Salinet J, Paredes JG, Mazzetto M, Marques VG, Samesima N, Pastore CA, Cestari IA. Non-invasive characterization of cardiac activation patterns: Contributions of body surface potential mapping in healthy volunteers. In 2019 Computing in Cardiology (CinC). IEEE, 2019; 1-4.

[17] van Oosterom A, Ihara Z, Jacquemet V, Hoekema R. Vectorcardiographic lead systems for the characterization of atrial fibrillation. J Electrocardiol 2007;40(4):343-e1.

Address for correspondence:

Victor Gonçalves Marques

Biomedical Engineering - CECS

Federal University of $\mathrm{ABC}$

E-mail: vgmarques.ufabc@gmail.com 